\title{
Transperineal ultrasound to assess anal positioning in female neonates
}

\author{
Mathilde Casson-Masselin ${ }^{1}$, Didier Moriau ${ }^{1}$, Claire Bahans ${ }^{2}$, Grazia Spampinato ${ }^{3}$, Vincent \\ Guigonis $^{2}$, Quentin Ballouhey ${ }^{3}$, Laurent Fourcade ${ }^{3}$
}

${ }^{1}$ Radiology Department, ${ }^{2}$ Paediatrics Department, ${ }^{3}$ Paediatric Surgery Department, Limoges University Hospital Limoges, France

\begin{abstract}
Aims: Anterior anal displacement is a condition that is difficult to define: is it merely an anatomical variation within the range of normality or is it a genuine malformation? In order to improve early diagnosis of anterior displacement of the anus, this study assessed the feasibility of transperineal ultrasound (US) in routine practice for use as first-line management. Materials and methods: Sagittal perineal US was performed on 187 female infants aged between 3 and 9 weeks. The ultrasound was performed randomly, either by a senior or a junior radiologist. The imaging by the senior radiologists defined the sagittal plane quality criteria. Scores to define image quality were established and then validated. The percentage of satisfactory images was then compared according to the planes and the seniority (junior vs. senior) of the radiologists. The junior radiologists were evaluated from the beginning to determine the learning curve for optimal image acquisition. Results: The quality scores for the perineal ultrasounds revealed excellent intra-and inter-operator performances: $84.4 \%$ of the sagittal plane images were satisfactory. After the $20^{\text {th }}$ examination, the success rate for the sagittal plane was independent of the seniority of the radiologist. Conclusions: This preliminary study demonstrates that transperineal sagittal plane US is easy to learn and readily yields reproducible results. The possibility of using this technique provides a solid basis for further studies to evaluate its relevance in regard to clinical data and MRI results, with the aim of providing a reliable early diagnostic tool for routine practice.
\end{abstract}

Keywords: infant; anterior displacement of the anus; ultrasound; perineum; learning curve

\section{Introduction}

Anterior displacement of the anus (ADA) is a condition that affects between 5 and $10 \%$ of the population and it occurs predominantly in girls [1]. In practice, the condition is diagnosed by simply observing the perineum, either during the routine examination undertaken at birth (anal/genitalia position, perianal radial folds) or during subsequent consultations for the constipation occurrence during the first months of life or right after the introduc-

Received 09.06.2020 Accepted 16.09.2020

Med Ultrason

2021, Vol. 23, No 1, 36-41

Corresponding author: Quentin Ballouhey

Limoges University Hospital,

Department of Paediatric Surgery,

8 Avenue Dominique Larrey,

87000 Limoges, France

E-mail: q.ballouhey@gmail.com

Tel: +33 (0)5 55058753

Fax: +33(0)5 55056050 tion of solid food in the infant's diet. With ADA, the ano-vulvar distance becomes less and the perianal radial folds disappear to varying degrees. In 1984, Reisner et al [2] proposed a clinical index, known as the anal position index (API). This index measure relies on the position of the coccyx tip as a fixed landmark (fig 1). API is defined as the ratio of the anal-fourchette distance and the coccyx-fourchette distance for females. The threshold beneath which the anus is considered to be in the anterior position is a ratio of 0.34 for girls and 0.46 for boys [2].

The coccyx position and anatomy varies from person to person (fig 2), which renders this clinical index somewhat unreliable for the global assessment of the degree of anal anterior displacement. Ultrasound (US) sagittal plane images centered on the pelvis and performed strictly at the same level and in the same plane used for the API measurement, allow a global perineal study of the urethra, vagina, anal canal and anus, as well as the interface between the distal vagina and the anal canal [3]. 


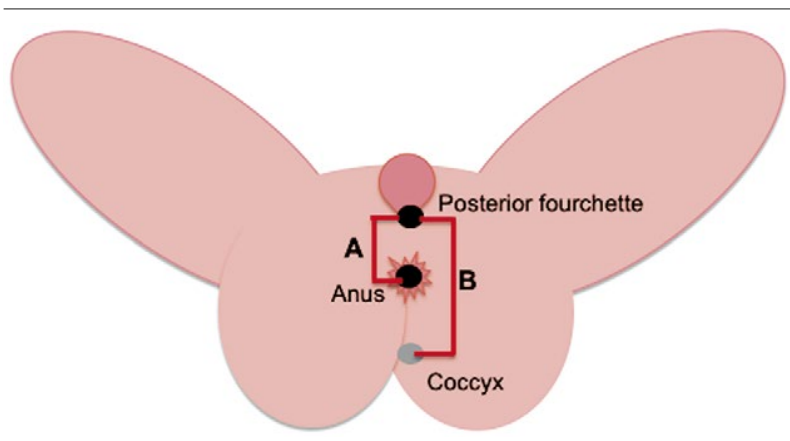

Fig 1. Index of the anal position for females. A: Fourchette - Anal distance; B: Fourchette - Coccyx distance; API $=\frac{\text { Fourchette-Anal distance }(\mathrm{cm})}{\text { Fourchette-Coccyx distance }(\mathrm{cm})}$

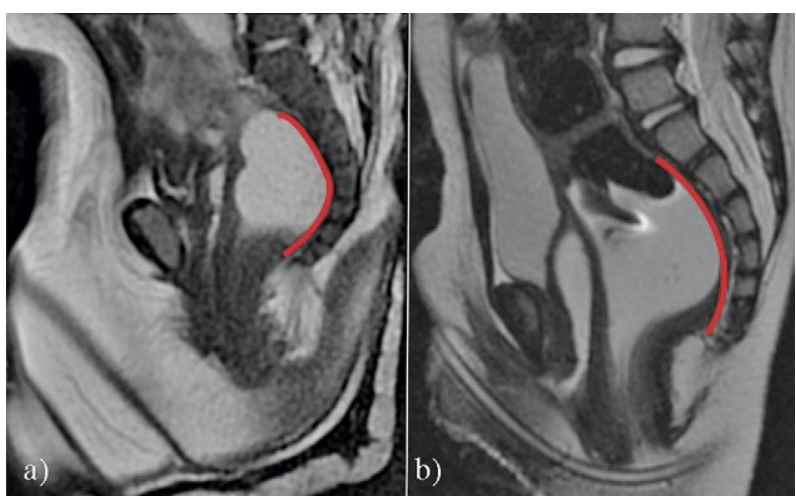

Fig 2. Illustration of the variable position of the coccyx tips with MRI. MRI sagittal images (T2 sequence) of two unaffected one-year old patients showing variation of the coccyx tip: a) the ventral curve of the coccyx tips (red line); b) the dorsal deflection of the coccyx tips (red line)

ADA can be the consequence of a simple defect in cloacal membrane caudal expansion, or it can reflect a more substantial underlying anatomical disorder [4]. It is now generally accepted that minor abnormalities at the external anal orifice are associated with deeper anatomical aberrations in boys [5]. With ADA in girls, US can reveal significant issues relating to the arrangement of the digestive and genital compartments, which to varying degrees involve the sphincter complex, resulting in: verticalization of the anal canal, with disappearance of the anorectal angle or even an anterior obliquity of the anal canal; reduction/disappearance of the perineal body, causing a similar significant reduction of the space between the anterior surface of the distal anal canal and the distal posterior vaginal wall (perineal body); modification of the sphincter complex; and pre-vestibular placement of the anus is to be considered to be the frontier with anorectal malformation.

There is, however, no correlation between the data obtained at the clinical examination performed in the first days of life for ADA and the gradual onset of severe constipation [6-8]. Prognostic criteria would help physicians in deciding whether to merely engage in medical monitoring or whether early surgery would, in fact, be beneficial. Many authors tend to consider ADA to be a heterogeneous disease, with an anatomical continuum for which the extreme forms vary from the simple approximation of the anus towards the vulva to anorectal malformation with the anus in a pre-vestibular position $[9,10]$.

Only a limited number of complementary investigations are available to surgeons to assess the seriousness of the anatomical manifestation of this condition during the early months of life. MRI and manometric tests are used in older children. Perineal US is already being used for various congenital or acquired anomalies in infants [3], including anorectal malformations.

In this context, it can be hypothesized that perineal sagittal US could help with investigation of the deep perineal anatomical structures and their relative arrangement as visualized by MRI [11], in agreement with the medical examination.

There is currently no information regarding the feasibility or reproducibility of US for this indication. The aim of this study was, hence, to investigate the feasibility and reproducibility of acquiring US sagittal plane images in girls during the first 3 months of life.

\section{Materials and methods}

This prospective, observational, single-center study was carried out by six radiologists $(2$ senior and 4 residents) in the Pediatric Radiology Department of our University Hospital between July 2017 and June 2018. The study protocol was approved by our administrative region's Ethics Committee. The parents were provided written information about the study in the waiting room and their consent was obtained before the ultrasound was performed. They were also present throughout the examination.

The proposed measurement should provide an estimate of the distance between the middle of the distal urethra and the middle of the anus in girls without an anomaly, thereby defining a standard "ultrasound API". The study was, therefore, carried out on girls, who systematically undergo a routine hip ultrasound for screening of congenital hip dysplasia during the first month of life in our center. The criteria for inclusion comprised, female without preterm birth or associated malformation and aged between 3 and 9 weeks. The exclusion criteria were: age less than 3 weeks or more than 9 weeks, male, or parents' refusal. The age, weight, and height of each infant were recorded. Perineal ultrasound [Super- 


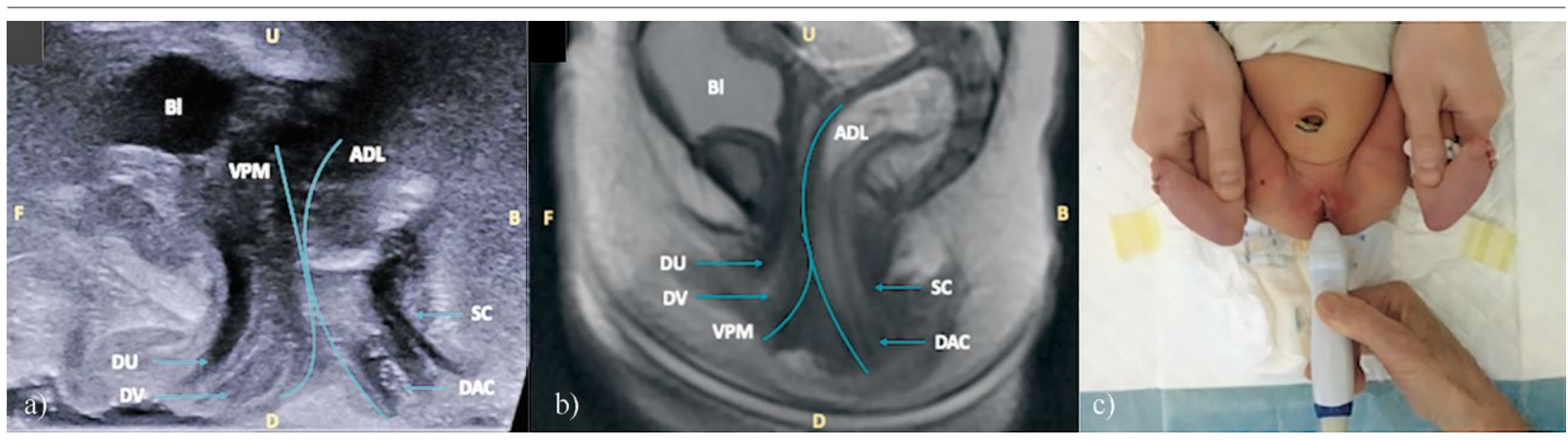

Fig 3. Sagittal plane images comparing the US (a) and the MRI (b) modality on the same patient. The US modality (a) can provide comparable information to MRI (b) for deep perineal landmarks in girls. Bl: bladder; VPM: vagina posterior margin; ADL: anterior digestive limit; DU: distal urethra; DV: distal vagina; SC: sphincter complex; DAC: distal anal canal; U: up; D: down (perineal surface); F: front; B: back; (c) Infant positioning during the US examination: the infant was placed in dorsal decubitus with their legs folded over the abdomen. The probe, with at least $5 \mathrm{~mm}$ of US gel, was placed, without exerting any pressure, on the infant's perineum, in the sagittal position.

sonic ImagineAixplorer Ultimate ${ }^{\circledR}$ (Aix-en-Provence, France) machine, $15-4 \mathrm{MHz}$ SuperLinear ${ }^{\mathrm{TM}}$ probe] was performed at the same time. The mean total duration of the examination was 10 minutes and it was very well tolerated by the patients.

Image acquisition

A review of the literature enabled us to identify the perineum plane of interest $[3,10]$. Concerning the normal aspect of the sagittal plane, the US modality (fig 3a) can provide comparable information to MRI (fig 3b). The infant was placed in dorsal decubitus with their legs folded over the abdomen (fig 3c). The patient needs to lie still and a large quantity of gel should be used. The operator placed at least $5 \mathrm{~mm}$ of ultrasound gel onto the probe, which was then placed, without exerting any pressure, onto the infant's perineum, in the sagittal position. The image was stored on the computer hard drive. The sagittal plane was defined by visualization of the pubic symphysis and the urethra on the same image. This made it possible to visualize the vagina and the digestive compartment in girls.

Either a radiology resident in the first semester of pediatric radiology, with no prior experience with perineal US, or a senior radiologist with at least ten years' experience, performed the US examination on each consecutive patient. The four junior radiologists were trained in two stages: a theoretical presentation of the technique by one of the two senior radiologists using slides, followed by a practical lesson that involved assisting a senior radiologist during performing three examinations. The junior radiologists then carried out the US examinations without supervision.

\section{Defining quality scores}

The criteria for the image quality were defined beforehand by senior radiologists (SR1, SR2) using the quality criteria defined by Son et al [3] and Hosokawaet al [12]: transperineal US was performed with a high-frequency linear array transducer $(8 \mathrm{MHz}$ or higher), with a thick coating of gel over the perineum to eliminate air artifacts. The midsagittal plane was identified by the presence of the pubic symphysis and urethra on the same image. The vagina was also visualized between the rectum and the bladder.

The scoring involved six criteria (Table I) based on the presence or absence of major and minor quality criteria, thereby allowing definition of an overall score (0-9). By convention, scores above 4 were considered to be satisfactory. The scoring was based on Herman's image-scoring method [13] for US screening of prenatal trisomy 21 .

\section{Score validation}

The US images taken by the junior (JR1, JR2, JR3, and JR4) and the senior radiologists (SR1 and SR2) were stored using designated computer software and then reviewed separately and independently after a blinding procedure by SR1 and SR2. Each of them allocated a quality score. In case there was any doubt whether the quality of an image was satisfactory, it was deemed to

Table I. Quality criteria of ultrasound images

\begin{tabular}{|c|c|}
\hline \multicolumn{2}{|l|}{ Quality criteria of sagittal plane } \\
\hline Major criteria (2 points) & Minor criteria ( 1 point $)$ \\
\hline $\begin{array}{l}\text { Strictly sagittal plane visualizing } \\
\text { the three compartments* } \\
\text { and the full length of the urethra }\end{array}$ & $\begin{array}{l}\text { Axis of the urethra } \\
\text { in the anterior third } \\
\text { of the image }\end{array}$ \\
\hline $\begin{array}{l}\text { Axis of the urethra perpendicu- } \\
\text { lar to the probe plane }\end{array}$ & $\begin{array}{l}\text { Probe focus on the } \\
\text { anal canal }\end{array}$ \\
\hline $\begin{array}{l}\text { Gel interface visible between } \\
\text { the probe and the skin }\end{array}$ & $\begin{array}{l}\text { Gain enabling visualiza- } \\
\text { tion of the stratified aspect } \\
\text { of the vaginal walls }\end{array}$ \\
\hline
\end{tabular}

* urinary, genital, and digestive compartments 
be unsatisfactory. Scoring of all of the images allowed us to compare the extent to which the scores of these two reviewers were in agreement.

\section{Analysis of the results obtained by the junior radiologists}

The number of satisfactory images taken by the junior radiologists and by the senior radiologists was determined. The percentage of satisfactory images was compared between the operator groups, and the percentage for the senior radiologists was taken as the reference. The learning curves of the junior radiologists were established in order to determine the threshold for the number of examinations required in order to reach the standard of the senior radiologists.

\section{Statistical analysis}

The statistical analyses for this study were performed using Excel 2010 ${ }^{\circledR}$ software (Microsoft Corporation, Redmond, WA, USA) and R Statistical Software (version 3.1.3). A p-value below 0.05 was considered to be statistically significant. Comparisons were carried out using the Mann-Whitney test for the quantitative variables, the $\mathrm{Chi}^{2}$ test or Fisher's exact test (if a small sample size for comparison of the qualitative variables), and the Wilcoxon signed-rank test for the paired data. The concordance between the scores of the two senior reviewers was assessed using a Bland-Altman plot with 95\% limits of agreement for each comparison (average difference \pm 2 standard deviations (SD) of the difference).

\section{Results}

\section{Description of the study populations}

During the study period, 187 infant girls were included in the study. Their mean age was $6.6 \pm 1.0$ weeks, their mean weight was $4.080 \pm 640 \mathrm{~g}$, and their mean height was $53 \pm 3 \mathrm{~cm}$.

The senior radiologists performed 94 ultrasound examinations $(\mathrm{SR} 1=71, \mathrm{SR} 2=23)$, and the junior radiologists performed 93 ultrasound examinations $(\mathrm{JR} 1=7$, $\mathrm{JR} 2=41, \mathrm{~J} 3=35, \mathrm{~J} 4=10$ ). The patient characteristics were similar in both radiologist groups.

\section{Scoring: inter-operator variability}

The inter-operator variability analysis did not reveal any statistically significant difference between the senior radiologists' scores ( $n=187$; SR1: 6.3 \pm 2.3 ; SR2: 6.1 \pm 2.5 , $\mathrm{p}=0.91$ ). The Bland-Altman plots show the high level of agreement between the senior radiologists (fig 4).

\section{Intra-operator variability}

The intra-operator variability analysis did not reveal any statistically significant difference $(p=0.72)$ between the allocated scores for the ten examinations reviewed twice by the same senior radiologist, whether SR1 or SR2.

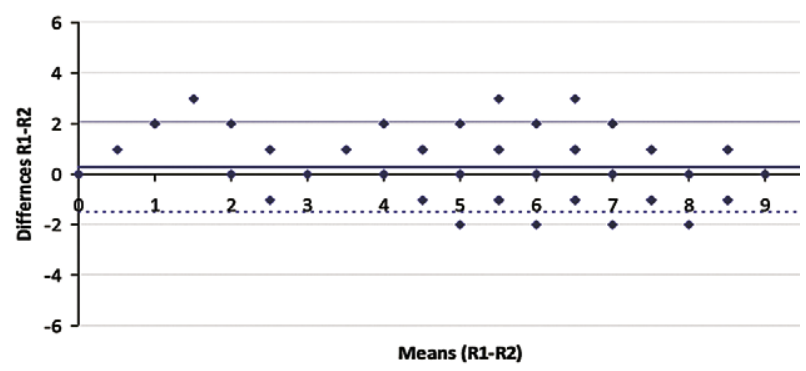

Fig 4. A Bland-Altman plot of the agreement in the scores between the two senior radiologists (SR1 and SR2). The central line corresponds to the mean difference in the scoring between the two senior radiologists (SR1 and SR2), the upper and lower dashed lines represent \pm 2 standard deviations. The graph shows the high level of concordance between the SR1 scores and the SR2 scores for the sagittal plane images.

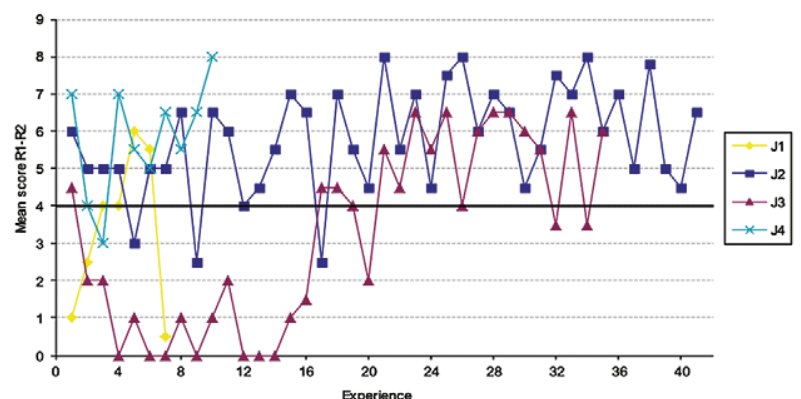

Fig 5. The learning curve for the junior radiologists - JR1, JR2, JR3, and JR4. The scores of the four junior radiologists are shown according to their acquisition of experience. The learning plateau is situated immediately after the $20^{\text {th }}$ examination.

\section{Percentage of ultrasound examinations retained according to the radiologist status}

Based on the quality score (defined in Table I), $84.5 \%$ of the sagittal images (158/187) were deemed to be satisfactory. Moreover, the rates of ultrasound examinations retained differed according to the seniority (junior vs. senior) of the radiologists: for the junior radiologists, 67 images $(72 \%)$ of the 93 that were taken were satisfactory, vs. 91 images $(97 \%, \mathrm{p}<0.0001)$ of the 94 that were taken by the senior radiologists.

The learning curves for sagittal plane transperineal ultrasound showed that the two junior radiologists with an experience of more than 10 imaging procedures exhibited a plateau of satisfactory images after the $20^{\text {th }}$ examination (fig 5).

\section{Discussion}

Our study demonstrates that sagittal plane US imaging is feasible and reproducible in routine practice. Early diagnosis of ADA is based on straight forward clinical observation of the child's perineum, while checking for 
any anterior displacement, coccygeal dimple or an anomaly of the radial folds. The only objective clinical criterion available is Reisner's anal position index (API) [2], the merits of which remain highly debatable [11]. Perineal US is prescribed for children with anorectal malformations with imperforate anus in order to determine the distance to the skin of the anal sac and to determine the location of the fistula. The simple transperineal US technique for visualizing the main perineal problems has been described extensively [3,14-18].

Certain imaging centers, including ours, perform perineal US for any clinical abnormality in the anal position, although the relevance of this technique remains marginal since its reproducibility and feasibility for routine practice are not known. In addition to this clinical diagnosis, the tools currently available for surgeons also include manometric tests [19] and MRI. MRI is increasingly being used and it can reveal the severity of the ADA. It can identify an abnormal orientation of the lower part of the anal canal, positioned towards the front. Therefore, it allows for a loco-regional anatomic evaluation [20, 21].

We proposed standardization of sagittal transperineal ultrasound examination of infant girls by the implementation of a quality score. This score is based on the use of simple criteria to improve the reproducibility of images. Determination of the criteria to define ultrasound image quality is crucial for the practice of transperineal US in children [3]. The quality criteria applied to the planes ensured strictly sagittal planes, the absence of substantial pressure being exerted on the child's perineum, good probe positioning and correct parameter settings. It is particularly important to place the probe at a right angle to the perineum to obtain the urethra ventrally to the digestive compartment but on the same image thanks to a good depth of focus. No difficulties were encountered relating to the patient's movements. In our opinion, the existence of such criteria ensured that the US images were very close to the morphological analysis obtained by sagittal T2-weighted MRI.

We found no evidence of intra- and inter-operator variability, thereby providing further support for these criteria. Moreover, the analysis of the success rates of satisfactory images for the junior and the senior radiologists revealed a statistically higher percentage of success for the senior radiologists, which is further indication of the relevance of the selected quality criteria. The percentage of satisfactory images was above $80 \%$ and the percentage of success for the junior radiologists was $72 \%$. As indicated by the learning curve, their success in this regard was the same as that of the senior radiologists by the time the $20^{\text {th }}$ examination had been carried out. Junior radiologists can readily reach this 20 -examination threshold in routine practice as this can be achieved in one-day training sessions. Furthermore, this threshold is consistent with the learning curves in other studies that assessed ultrasound training for inexperienced operators [22,23].

ADA is a condition that is difficult to define, as indicated by the diverse terminology in the medical literature. For example, "anterior ectopic anus", "anteriorly displaced anus", "anterior perineal anus", "rectoperineal fistula", are all terms used to describe this condition[24], and they refer to the abnormal anterior position of the anus in the perineum. Does it represent an anatomical variation within the range of normality, or is it a genuine malformation configured as an ARM?

The first step of this US acquisition is to exclude a digestive fistula. However, the present approach was aimed more at proposing a radiological approach of the particular condition of ADA than to achieve MAR subtype definitions. The present study shows the importance of training and familiarisation for ultrasound operators lacking ultrasound experience using standardized procedures. The fact that this technique can be learned quickly and used by different operators opens up new perspectives, both for radiologists and for surgeons. A sagittal plane ultrasound of the perineum can be performed in routine practice to obtain reproducible and reliable images of ADA or anorectal malformations. Thus, other parameters such as the orientation of the lower part of the anal canal and the size of the perineal body could be considered in further studies. The possibility of using routine perineal ultrasound could then lead to definition of an "ultrasound API", which may or may not correlate with clinical measurements, and which would also enable screening of the deep anatomical changes reported in severe forms of anal displacement that are similar to anorectal malformations.

The main limit of this study is the absence of systematic reference imaging with MRI modality.

\section{Conclusions}

In conclusion, this study shows the importance of training for operators who lack US experience using standardized procedures in order to assist them with their clinical examinations. The sagittal plane acquisition technique can be learned quickly and it can be used by different practitioners, thereby opening up new perspectives. Due to the lack of clinical data and reliable complementary examinations at birth, certain forms of ADA are difficult to classify. By providing better imaging of the anatomical abnormalities, perineal US could be of assistance in the management of ADA. The possibility of 
using this technique provides a solid precedent for further studies to evaluate its relevance to clinical data and MRI results, with the aim of providing a reliable early diagnostic tool for routine practice.

\section{References}

1. Genç A, Taneli C, Tansuğ N, et al. Evaluation of the location of the anus by a modified technique in the neonate. $\mathrm{J}$ Pediatr Surg 2002;37:80-82.

2. Reisner SH, Sivan Y, Nitzan M, Merlob P. Determination of anterior displacement of the anus in newborn infants and children. Pediatrics 1984;73:216-217.

3. Son JK, Taylor GA. Transperineal ultrasonography. Pediatr Radiol 2014;44:193-201.

4. Kluth D, Fiegel HC, Metzger R. Embryology of the hindgut. Semin Pediatr Surg 2011;20:152-160.

5. AbouZeid AA, Mohammad SA. Low-type anorectal malformations in the male: Extent of deviation from the norm. J Pediatr Surg 2016;51:1851-1858.

6. Leape LL, Ramenofsky ML. Anterior ectopic anus: a common cause of constipation in children. J Pediatr Surg 1978;13:627-630.

7. Herek O, Polat A. Incidence of anterior displacement of the anus and its relationship to constipation in children. Surg Today 2004;34:190-192.

8. Núñez-Ramos R, Fabbro MA, González-Velasco M, et al. Determination of the anal position in newborns and in children with chronic constipation: comparative study in two European healthcare centres. Pediatr Surg Int 2011;27:1111-1115.

9. Herek O. Anterior ectopic anus: an accurate definitive term for choice of treatment? Pediatr Surg Int 2001;17:501.

10. Peña A. Comments on anterior ectopic anus. Pediatr Surg Int 2004;20:902.

11. AbouZeid AA, Mohammad SA, Khairy KT. MRI anatomy of anteriorly displaced anus: what obstructs defecation? Pediatr Radiol 2014;44:831-838.

12. Hosokawa T, Yamada Y, Hsokawa M, et al. Ultrasound imaging of the anorectal malformation during the neonatal period: a comprehensive review. Jpn J Radiol 2018;36:581591.

13. Herman A, Maymon R, Dreazen E, Caspi E, Bukovsky I, Weinraub Z. Nuchal translucency audit: a novel imagescoring method. Ultrasound Obstet Gynecol 1998;12:398403.

14. Cremin BJ. A review of the ultrasonic appearances of posterior urethral valve and ureteroceles. Pediatr Radiol 1986; 16:357-364.

15. Cohen HL, Susman M, Haller JO, Glassberg KI, Shapiro MA, Zinn DL. Posterior urethral valve: transperineal US for imaging and diagnosis in male infants. Radiology 1994;19:261-264.

16. Donaldson JS, Black CT, Reynolds M, Sherman JO, Shkolnik A. Ultrasound of the distal pouch in infants with imperforate anus. J Pediatr Surg 1989;24:465-468.

17. Teele RL, Share JC. Transperineal sonography in children. AJR Am J Roentgenol 1997;168:1263-1267.

18. Paltiel HJ, Phelps A. US of the pediatric female pelvis. Radiology 2014;270:644-657.

19. Schuster T, Joppich I, Schneider K, Jobst G. A computerised vector manometry study of the so-called ectopic anus. Pediatr Surg Int 2000;16:8-14.

20. Van Beers BE, Kartheuser A, Delos MA, et al. MRI of the anal canal: correlation with histologic examination. Magn Reson Imaging 1996;14:151-156.

21. Ballouhey Q, Fourcade L, Durand-Fontanier S, et al. Variations anatomiques de l'anus et constipation chez le nouveau-né. Quelle contribution de l'IRM ? Morphologie 2014;98:107-108.

22. Gracias VH, Frankel HL, Gupta R, et al. Defining the learning curve for the Focused Abdominal Sonogram for Trauma (FAST) examination: implications for credentialing. Am Surg 2001;67:364-368.

23. Burford JM, Dassinger MS, Smith SD. Surgeon-performed ultrasound as a diagnostic tool in appendicitis. J Pediatr Surg 2011;46:1115-1120.

24. Pakarinen MP, Rintala RJ. Management and outcome of low anorectal malformations. Pediatr Surg Int 2010;26:10571063. 